\title{
The synthesis of seismic signals for laterally inhomogeneous geological media via BIEM
}

\author{
I.-K. Fontara ${ }^{1}$, S. L. Parvanova ${ }^{2}$, P. S. Dineva ${ }^{3}$, T. Rangelov ${ }^{3}$ \\ \& F. Wuttke ${ }^{1}$ \\ ${ }^{1}$ Institute of Applied Geoscience, \\ Christian-Albrechts-University of Kiel, Germany \\ ${ }^{2}$ University of Architecture, Civil Engineering and Geodesy, Bulgaria \\ ${ }^{3}$ Bulgarian Academy of Sciences (BAS), Bulgaria
}

\begin{abstract}
Two-dimensional mechanical models consisting of discrete and continuous systems, accompanied with different boundary integral equation (BIE) techniques, are used for the synthesis of seismograms in a laterally inhomogeneous geological profile situated in a half-plane which mechanical properties vary with respect to the depth. Seismic load is time-harmonic, presented by incident SH-wave or wave radiating from an embedded line seismic source. The material inhomogeneity is modelled in two different ways: (i) as a continuous model where material properties are quadratic functions of depth; (ii) as a discrete model where material properties vary in a discrete way and the geological profile is presented by a stack of flat horizontal layers. The computational tools are based on the BIEM using: (i) analytically derived Green's function for a quadratically inhomogeneous in depth half-plane; (ii) a hybrid (wave number integration method) WNIMBIEM; or (iii) direct displacement BIEM based on the elastodynamic fundamental solution for the full plane. A validation study is presented. All simulations reveal the sensitivity of the synthetic seismic signals on the type and characteristics of the seismic load, on the wave path inhomogeneity and on the geotechnical properties of the local geological region as sub- and free- surface relief and material gradient with respect to the depth.
\end{abstract}

Keywords: SH-wave, relief, Green's function for inhomogeneous half-plane, BIEM, synthetic seismograms. 


\section{Introduction}

The development of an efficient tool for seismic signal synthesis is based on the appropriate choice of adequate model describing the seismic source, the wave path and the local geological region of interest. The developed methods of analysis can be briefly classified as: analytical [1], numerical [2] and hybrid methods [3, 4]. Among various numerical methods used, the BEM seems to be the most effective for the discussed problems due to its well-known advantages over the domain approach, see Dominguez [2]. To the authors' best knowledge there are limited BEM codes for modelling of the whole solid Earth system consisting of the seismic source, wave path and local region of interest.

In the present paper two-dimensional mechanical models continuous and discrete accompanied with three different BIEM techniques, able to account for all three components of the Earth system, are presented. The continuous model in which the material properties vary as a continuous function with respect to the depth is solved by a non-conventional BIEM based on the Green's function for quadratically inhomogeneous half-plane. The discrete model describes the inhomogeneity in depth by a stack of flat horizontal homogeneous layers and it is treated by a hybrid WNIM-BIEM or by a pure BIEM based on the frequencydependent elastodynamic fundamental solution for the full plane.

\section{Problem statement}

Consider elastic isotropic inhomogeneous in depth half-plane with free surface relief of arbitrary shape subjected to: (i) time-harmonic incident $\mathrm{SH}$-wave or (ii) $\mathrm{SH}$-wave generated by an embedded line time-harmonic seismic source. Antiplane wave motion in plane $\mathrm{x}_{3}=0$ is assumed. The material inhomogeneity in depth is modelled by two different ways: (1) continuous model (Fig. 1a) where material properties are continuous quadratic functions of depth $\mu\left(x_{2}\right)=\mu_{0}\left(b_{2}+1\right)^{2}$ and $\rho\left(x_{2}\right)=\rho_{0}\left(b_{2}+1\right)^{2}$, where $b$ is the inhomogeneity parameter and $\mu_{0}, \rho_{0}$ the reference material properties, (2) discrete model (Fig. 1b) where the inhomogeneity is presented by a stack of flat horizontal layers, which are elastic, isotropic and homogeneous. Let us denote the boundary of the surface irregularity with $\mathrm{S}_{1}$ and the boundary $\mathrm{S}_{2}$ is the part of line out of the irregularity. The only nonzero field quantities are displacement $u_{3}(\mathbf{x}, \omega)$ and stresses $\sigma_{\mathrm{i} 3}(\mathbf{x}, \omega), \mathrm{i}=1,2, \mathbf{x}=\mathrm{x}\left(\mathrm{x}_{1}, \mathrm{x} 2\right)$. The governing equation of anti-plane timeharmonic wave motion due to incident plane $\mathrm{SH}$ wave or wave radiating by an embedded line seismic source in a point $\mathbf{x}_{0}$ is given by Eq. 1a for the continuous model and Eq. (1b) for the discrete one:

$$
\begin{gathered}
\sigma_{i 3, i}(\mathbf{x}, \omega)=-\rho\left(x_{2}\right) \omega^{2} u_{3}(\mathbf{x}, \omega)-\psi f_{03} \delta\left(\mathbf{x}, \mathbf{x}_{\mathbf{0}}\right) \mathbf{x} \in \Omega_{0}, \\
\sigma_{i 3, i}^{(k)}(\mathbf{x}, \omega)=-\rho_{k} \omega^{2} u_{3}^{(k)}(\mathbf{x}, \omega)-\psi f_{03} \delta\left(\mathbf{x}, \mathbf{x}_{\mathbf{0}}\right) \mathbf{x} \in \Omega_{k}, \quad k=0, \ldots N
\end{gathered}
$$


where $\psi=0$ when the seismic load is presented by the incident wave and $\psi=1$, when the seismic load is a wave radiating from an embedded line seismic source with magnitude $\mathrm{f}_{03}$, frequency $\omega$, and concentrated in a point $\mathbf{x}_{0}$. The wave propagating in the half-plane produces scattered wave when impinges on the freesurface relief in the continuous model and additionally on all interfaces in the discrete model. The total wave field is a superposition of the free-field motion $\left(\mathrm{u}_{3}{ }^{\mathrm{ff}}, \mathrm{t}_{3}{ }^{\mathrm{ff}}\right)$ and scattered wave field $\left(\mathrm{u}_{3}{ }^{\mathrm{sc}}, \mathrm{t}_{3}{ }^{\mathrm{sc}}\right)$, i.e. $\mathrm{u}_{3}=\mathrm{u}_{3}{ }^{\mathrm{ff}}+\mathrm{u}_{3}{ }^{\mathrm{sc}}$ and $\mathrm{t}_{3}=\mathrm{t}_{3}{ }^{\mathrm{ff}}+\mathrm{t}_{3}{ }^{\mathrm{sc}}$.
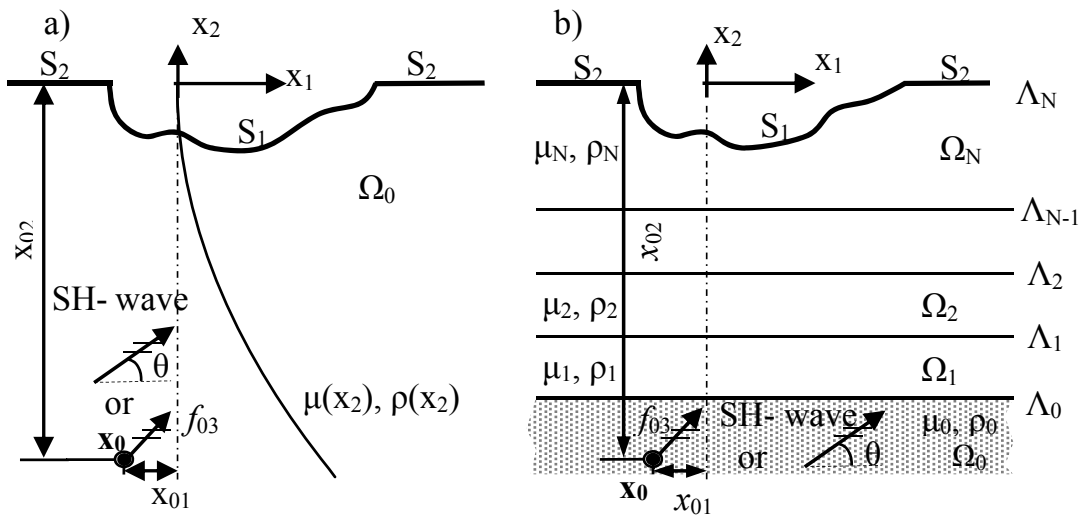

Figure 1: Geometry of the problem: (a) continuous, (b) discrete model.

The aim is solution of the defined problem for the displacement field, creation of the research software, its validation and usage for intensive simulations revealing the key role that material inhomogeneity and heterogeneity of a geological region play for synthesis of an adequate seismic signal.

The free-field motion in the continuous model describes wave propagation in a quadratically inhomogeneous in depth half-plane without any relief subjected to seismic load. The free-field motion under incident SH-wave produces the following displacement and traction at any observer point $\mathbf{x}$, see [5]:

$$
\begin{array}{r}
u_{3}^{f f}(\mathbf{x}, \omega)=u_{30}\left(b x_{2}+1\right)^{-1}\left[\frac{-b+i k \eta_{2}}{b+i k \eta_{2}} e^{i k<\mathbf{x}, \boldsymbol{\eta}_{-}>}+e^{i k<\mathbf{x}, \boldsymbol{\eta}>}\right] \\
t_{3}^{f f}=\mu(\mathbf{x}) u_{3, i}^{f f} n_{i}
\end{array}
$$

Here $\boldsymbol{\eta}=\left(\eta_{1}, \eta_{2}\right)=(\cos \theta, \sin \theta), \boldsymbol{\eta}_{-}=\left(\eta_{1},-\eta_{2}\right)=(\cos \theta,-\sin \theta),<\ldots, . .>$ is a scalar product, $k=\sqrt{\frac{\rho_{0}}{\mu_{0}}} \omega, u_{30}$ is the unit amplitude.

In the case of the discrete model the free-field motion concerns wave propagation in: (i) a horizontally stratified half-plane without any relief when we 
use the hybrid WNIM-BEM and the numerical tool for solution of the free-field problem is analytical WNIM [4]; (ii) a homogeneous half-plane without any relief when we apply the pure BEM based on the fundamental solution for the full plane and here analytical solution for free-field motion is known [2].

What follows is the BIEM formulation of the considered problem for continuous and discrete model defined above when the seismic load is incident time-harmonic SH-wave or SH wave radiating from an embedded in the half-plane seismic source.

\section{BIEM formulation and solution}

\subsection{Continuous model with BIEM formulation based on the Green's function for quadratically inhomogeneous half-plane}

The scattered wave displacement along the free-surface boundary $S_{1}$, when the seismic load is incident $\mathrm{SH}$ wave propagating under incident angle $\theta$ can be found by Eq. (3):

$$
c u_{3}^{s c}(\mathbf{x}, \omega)=-\int_{S_{1}} t_{3}^{g^{*}}(\mathbf{x}, \xi, \omega) u_{3}^{s c}(\xi, \omega) d S_{1}-\int_{S_{1}} g_{3}^{*}(\mathbf{x}, \xi, \omega) t_{3}^{f f}(\xi, \omega) d S_{1}, \mathbf{x} \in S_{1}
$$

Here, $\mathbf{x}$ and $\boldsymbol{\xi}$ are the position-vectors of the source and field point respectively, $c$ is the jump term, $g_{3}$ is the Green's function for quadratically inhomogeneous in depth half-plane and $t_{3}^{g^{*}}$ is its corresponding traction, both derived analytically in [6]. The analytical derivation is based on the appropriate smooth transformation reducing the equation of motion (1a) with variable coefficients to one with constant coefficients with respect to the transformed function which solution is available. Once having solution for the transformed function we can obtain the original Green's function by the usage the inverse algebraic transformation [6]. Once having solution of Eq. (3), the total wave field is obtained as a sum of the scattered and free field motion quantities.

When the seismic load is wave radiating from an embedded seismic source located at a point $\mathbf{x}_{\mathbf{0}}$ in the inhomogeneous half-plane, the total wave displacement satisfies the following BIE:

$$
c u_{3}(\mathbf{x}, \omega)=-\int_{S_{1}} t_{3}^{g^{*}}(\mathbf{x}, \xi, \omega) u_{3}(\xi, \omega) d S_{1}+f_{03} g_{3}^{*}\left(\mathbf{x}, \mathbf{x}_{\mathbf{0}}\right) \mathbf{x} \in S_{1}
$$

Once having displacement along the boundary $\mathrm{S}_{1}$ we can find displacement and traction at any point in the half-plane by the well-known representation formulae. 


\subsection{Discrete model with BIEM formulation based on the fundamental solution for the full homogeneous plane}

Two computational BIEM tools based on the elastodynamic fundamental solution for the full plane are used: hybrid WNIM-BIEM and pure conventional displacement BIEM. To compute the total displacements at the canyon site by using the hybrid WNIM-BIEM proposed in [4], the following steps should be realized: (1) determine by WNIM the displacements and corresponding tractions for free-field motion along the points laying at the hypothetic canyon boundary $\mathrm{S}_{1}$; (2) apply tractions that are with the opposite sign of the tractions computed in step (1) at the base of the canyon as a traction boundary condition and calculate the scattered displacements along the free surface by the BIEM based on the elastodynamic fundamental solution for the full plane; (3) determine the total displacement by superposition formulae.

In the case when only the conventional displacement BIEM is applied, the following system of BIE with respect to the total wave field is satisfied when the seismic load is incident $\mathrm{SH}$ wave or wave radiated from the source:

$$
\begin{aligned}
& c u_{3}(\mathbf{x}, \omega)=\int_{S_{\Omega_{i}}} U_{3}^{*(i)}(\mathbf{x}, \mathbf{y}, \omega) t_{3}^{(i)}(\mathbf{y}, \omega) d S_{\Omega_{i}} \\
& -\int_{S_{\Omega_{i}}} P_{3}^{*(i)}(\mathbf{x}, \mathbf{y}, \omega) u_{3}^{(i)}(\mathbf{y}, \omega) d S_{\Omega_{i}}, \mathbf{x} \in S_{\Omega_{i}} ; i=1,2,3, \ldots N .
\end{aligned}
$$

Note, that the upper boundary of the layer $\Omega_{N}$ is $\Lambda_{N}=S_{1} \cup S_{2}$, where $S_{1}$ is the canyon's or hill's boundary, see Fig. 1. Remind that the following boundary condition is satisfied along the interface between the first layer and the seismic bed $t_{3}^{f f(0)}+t_{3}^{s c(0)}=t_{3}^{(0)}=-t_{3}^{(1)}$ for $\left(x_{1}, x_{2}\right) \in \Lambda_{0}$ where $t_{3}^{(0)}$ is the total traction in the region $\Omega_{0}$, while $t_{3}^{(1)}$ is the total traction at contour $\Lambda_{0}$ for layer $\Omega_{1}$. The total traction $t_{3}^{(0)}$, the free field traction $t_{3}^{f f(0)}$ and the free field displacement $u_{3}^{f f(0)}$ are all developed in the region $\Omega_{0}$ and are connected in the following BIE:

$$
\begin{aligned}
& c\left(u_{3}^{(0)}(\mathbf{x}, \omega)-u_{3}^{f f(0)}(\mathbf{x}, \omega)\right)=\int_{\Lambda_{0}} U_{3}^{*(0)}(\mathbf{x}, \mathbf{y}, \omega)\left(t_{3}^{(0)}(\mathbf{y}, \omega)-t_{3}^{f f(0)}(\mathbf{y}, \omega)\right) d \Lambda_{0}- \\
& -\int_{\Lambda_{0}} P_{3}^{*(0)}(\mathbf{x}, \mathbf{y}, \omega)\left(u_{3}^{(0)}(\mathbf{y}, \omega)-u_{3}^{f f(0)}(\mathbf{y}, \omega)\right) d \Lambda_{0} ; \mathbf{x} \in \Lambda_{0}
\end{aligned}
$$

In the case of embedded seismic source Eq. (6a) becomes: 


$$
\begin{aligned}
& c u_{3}^{(0)}(\mathbf{x}, \omega)=\int_{\Lambda_{0}} U_{3}^{*(0)}(\mathbf{x}, \mathbf{y}, \omega) t_{3}^{(0)}(\mathbf{y}, \omega) d S_{\Omega_{0}}- \\
& -\int_{\Lambda_{0}} P_{3}^{*(0)}(\mathbf{x}, \mathbf{y}, \omega) u_{3}^{(0)}(\mathbf{y}, \omega) d S_{\Omega_{0}}+f_{03} S(\omega) U_{3}^{*(0)}\left(\mathbf{x}, \mathbf{x}_{\mathbf{0}}\right) ; \mathbf{x} \in \Lambda_{0}
\end{aligned}
$$

\section{Illustrative examples and simulations}

The objective of the present section could be summarized as follows: (a) to demonstrate the equivalence of two different numerical models describing inhomogeneous in depth half-plane; (b) to reveal by simulations that the seismic field is a complex result of different key factors as the type and characteristics of the seismic load, the material inhomogeneity and the existence of free- and/or subsurface relief.

First we consider solution of the problem of continuously inhomogeneous halfplane modelled by continuous and discrete way, subjected to incident time harmonic SH- wave with incident angle $\theta$. The key study parameter is the amplification factor defined as the ratio of the displacement at the free-surface $\mathrm{x}_{2}=0$ to the displacement at the bottom $\mathrm{x}_{2}=-100$. The reference material properties are $\mu_{0}=180 \times 10^{6} \mathrm{~Pa}, \rho_{0}=2000 \mathrm{~kg} / \mathrm{m}^{3}$ and the inhomogeneity coefficient is chosen as $b=-1$. Fig. 2 demonstrates the equivalence between both mechanical models at fixed value of frequency $\mathrm{f}=1 \mathrm{~Hz}$ at different values of incident angle $\theta$, when the number of discrete layers increases. Fig. 3 illustrates the equivalence of the solutions at the free-surface obtained by the BIEM based on the Green's function for the continuous model and obtained by the hybrid WNIM-BIEM for the discrete model with number of layers $\mathrm{N}=100$. Fig. 3 concerns the case of inhomogeneous half-plane with semi-circular canyon with radius $a$ under normal incident $\mathrm{SH}$ - wave, for normalized inhomogeneity gradient $\beta=-\mathrm{b} /(2 a)$, reference material properties $\mu_{0}=180 \times 10^{6} \mathrm{~Pa}, \rho_{0}=2000 \mathrm{~kg} / \mathrm{m} 3$ and for frequency $\mathrm{f}=1 \mathrm{~Hz}$.

To the authors' best knowledge there are no results in the literature for quadratically inhomogeneous or homogeneous half-plane with different type of free-surface relief under $\mathrm{SH}$ - wave radiating from embedded seismic source. For this reason we validate our numerical scheme for homogeneous case with solutions obtained by conventional BIEM based on fundamental solution for the full plane. Consider homogeneous elastic isotropic half-plane with semi-circular canyon under $\mathrm{SH}$-wave radiating from embedded seismic source at point $(0,-5 a)$ and for seismic source magnitude $\mathrm{f}_{03}=10^{9} \mathrm{~N}$. Fig. 4 plots the surface displacement amplitude versus normalized distance at fixed values of normalized frequency $\eta=0.25$ and $\eta=1.0$, where $\eta=2 a / \lambda_{S H}$ ( $\lambda_{S H}$ is the wavelength). Observe that solutions obtained by BIEM based on the Green's function for the half-plane and on the fundamental solution for the full plane are almost identical. Fig. 5 reveals the sensitivity of the seismic field to the free-surface relief and to the material gradient. Fig. 6 shows the seismic signal along the free-surface when continuously inhomogeneous half-plane with inhomogeneity magnitude in the interval $0<\beta<0.05$ and with both semi-circle canyon and a circle cavity is considered at 
a fixed normalized frequency $\eta=1.25$ and a fixed seismic source magnitude $\mathrm{f}_{03}=10^{9} \mathrm{~N}$. Observe that the seismic signal changes its form when a circular cavity with diameter equal to $0.7 \mathrm{a}$ is added in the inhomogeneous half-plane. Fig. 7 shows the displacement wave field along the free surface of a stratified half-plane with a semi-elliptic canyon with semi-axes $a$ and $h$, subjected to wave radiating from a time-harmonic seismic source embedded at a point $(0,10 a)$. The used input data are: $\rho_{0}=\rho_{1}=\rho_{2}=\rho_{3}, \mu_{0}=10^{10} \mu_{3}, \mu_{1}=100 \mu_{3}, \mu_{2}=10 \mu_{3}, \mu_{3}$-shear modulus of the upper layer, the thickness of layers is $t=3 a$, and $h=\kappa a$ where $0 \leq \kappa \leq 2$. The normalization is done by the maximal displacement in the considered frequency range.

(a)

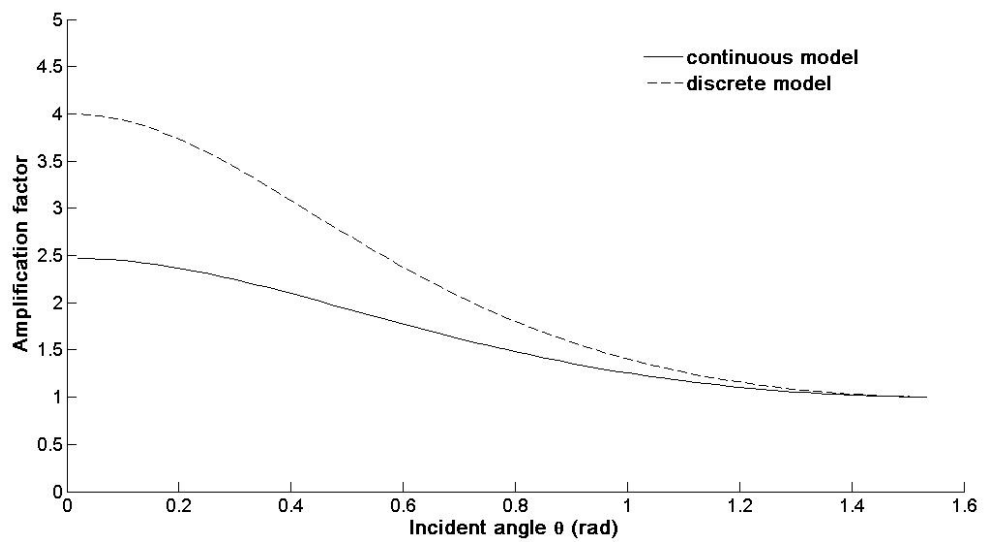

(b)

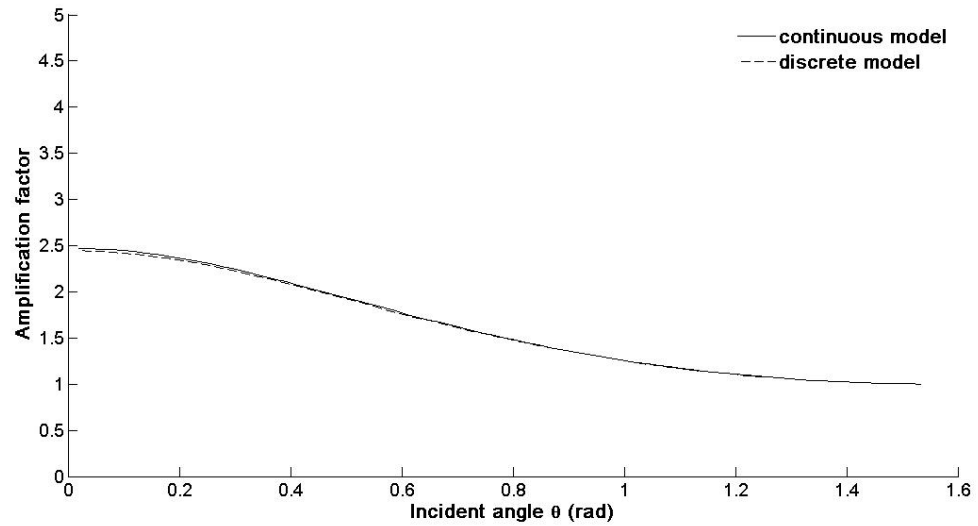

Figure 2: $\quad$ Amplification factor at $\mathrm{f}=1 \mathrm{~Hz}$ versus incident angle for the case of inhomogeneous in depth half-plane with inhomogeneous coefficient $\mathrm{b}=-1$ computed by the continuous and discrete model: (a) discrete model with 2 layers and (b) discrete model with 100 layers. 


\section{Conclusions}

Two dimensional elastodynamic problem is solved for $\mathrm{SH}$-wave propagation in inhomogeneous in depth half-plane with free or/and sub-surface relief of arbitrary shape and subjected to incident wave or wave radiating by an embedded seismic source. Two different models describing the variation of material characteristics with respect to the depth are considered: (a) continuous model where the soil characteristics are quadratic functions with respect to the depth and (b) discrete model presenting the half-plane as a stratum with homogeneous elastic isotropic horizontal layers. Three different computational tools based on the BIEM numerical scheme are applied: (a) for the continuous model, with quadratically inhomogeneous half-plane, BIEM based on Green's function is proposed, and (b) for the discrete model, hybrid WNIM-BIEM and conventional BIEM is employed.

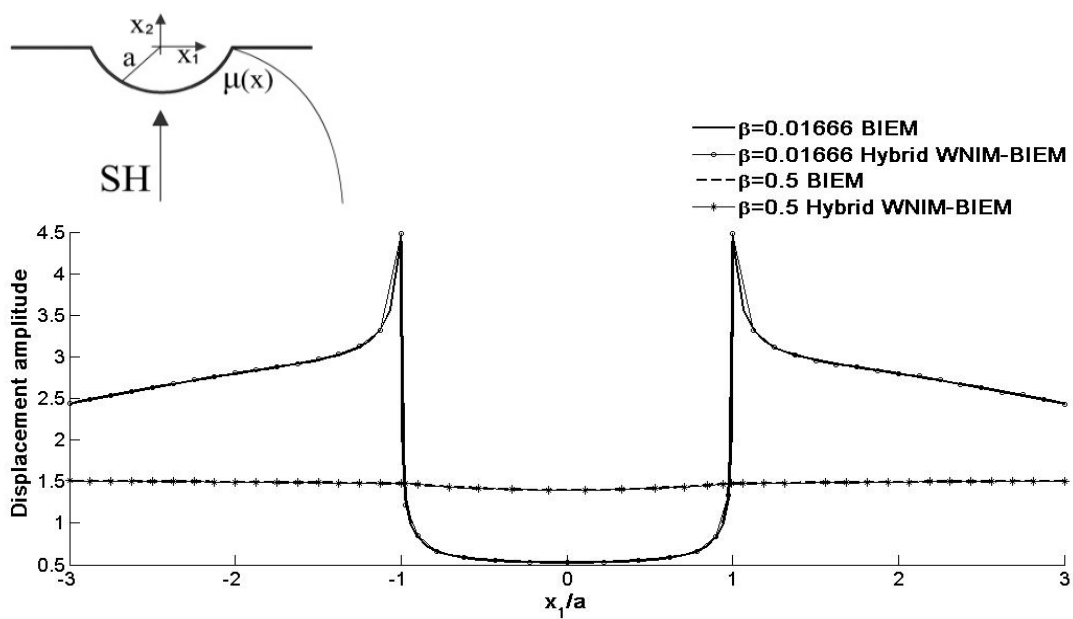

Figure 3: Displacement amplitude along the free-surface of quadratically inhomogeneous in depth half-plane. Comparison of solutions for continuous and discrete models at $\mathrm{f}=1 \mathrm{~Hz}$.

A comparison between these two mechanical models and the accompanied computational techniques reveals the advantages of the continuous model and the corresponding BIEM based on Green's function for the half-plane. In particular the following advantages are mentioned: (a) only the free- or sub-surface relief is discretised and thus avoiding discretization of all interfaces; (b) when hybrid technique is used, the backscattering phenomena are not taken into consideration and this is overcome by the continuous model; (c) the derived in a closed form Green's function has not a complex from and can be easily inserted in the BEM software frame. 

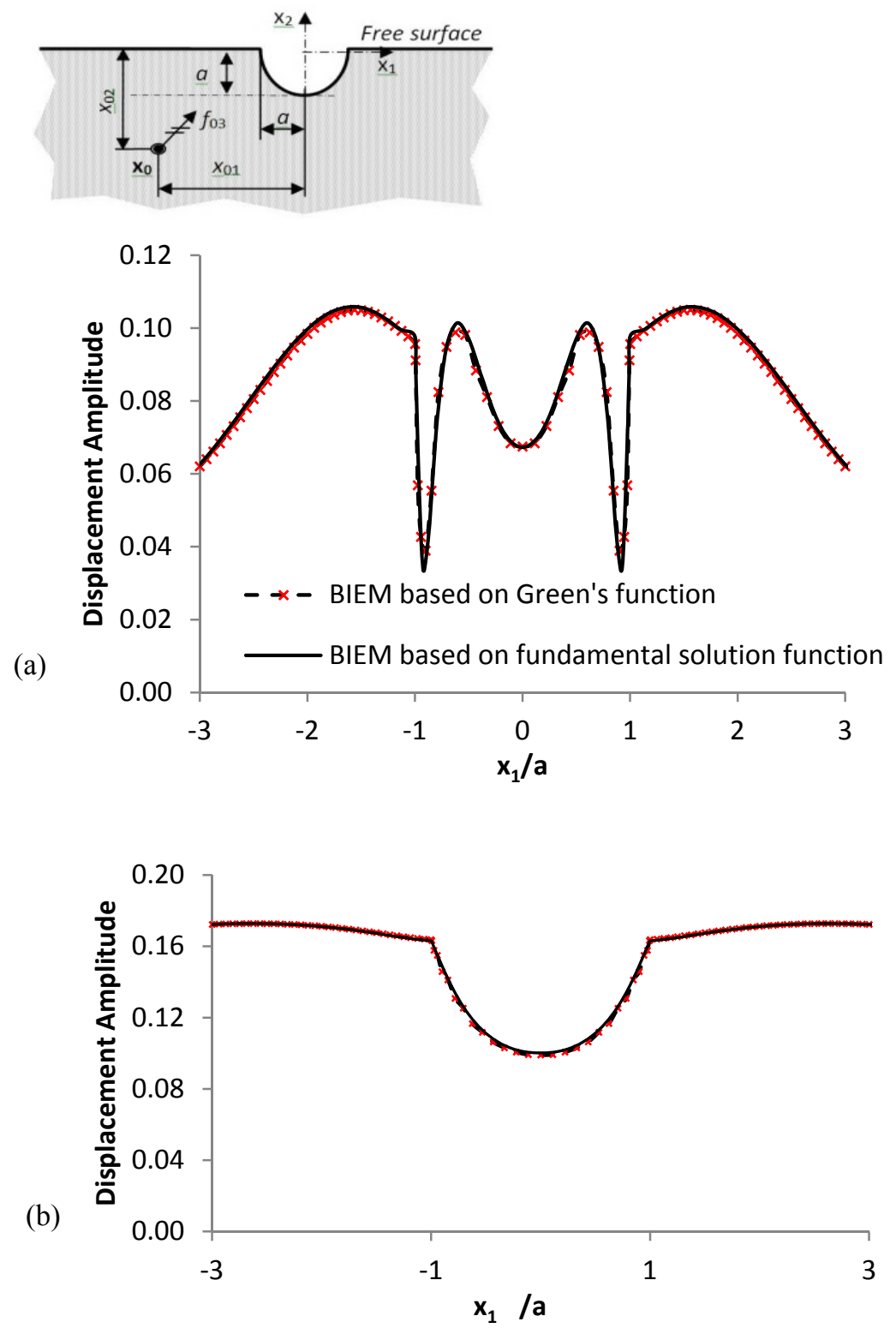

Figure 4: Displacement amplitude along the free surface of elastic homogeneous half-plane with a semi-circle canyon subjected to $\mathrm{SH}$ waves radiating from an embedded time-harmonic seismic source at point $(0,-5 a)$ for: a) $\eta=0.25, b) \eta=1.00$. Comparison between BIEM based on fundamental solution for the full plane and BIEM based on Green's function for homogeneous half-plane. 

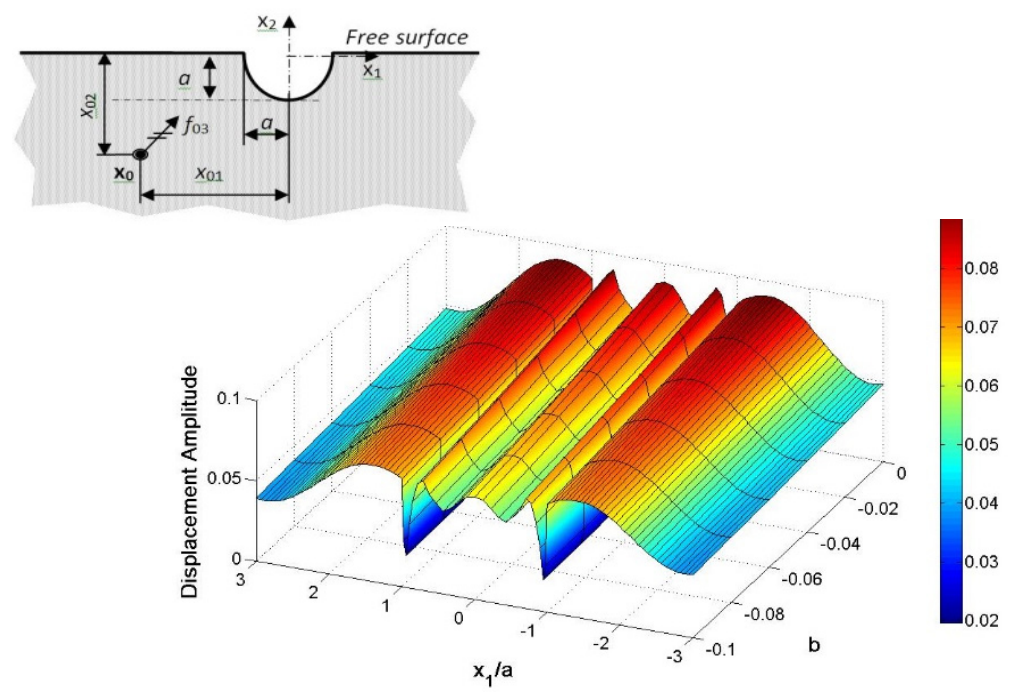

Figure 5: Displacement amplitude along the free surface of inhomogeneous half-plane with a semi-circular canyon at $0<\mathrm{b}<-0.1$ subjected to waves radiating from an embedded at point $(0,-5 a)$ seismic source with $\eta=1.25$.
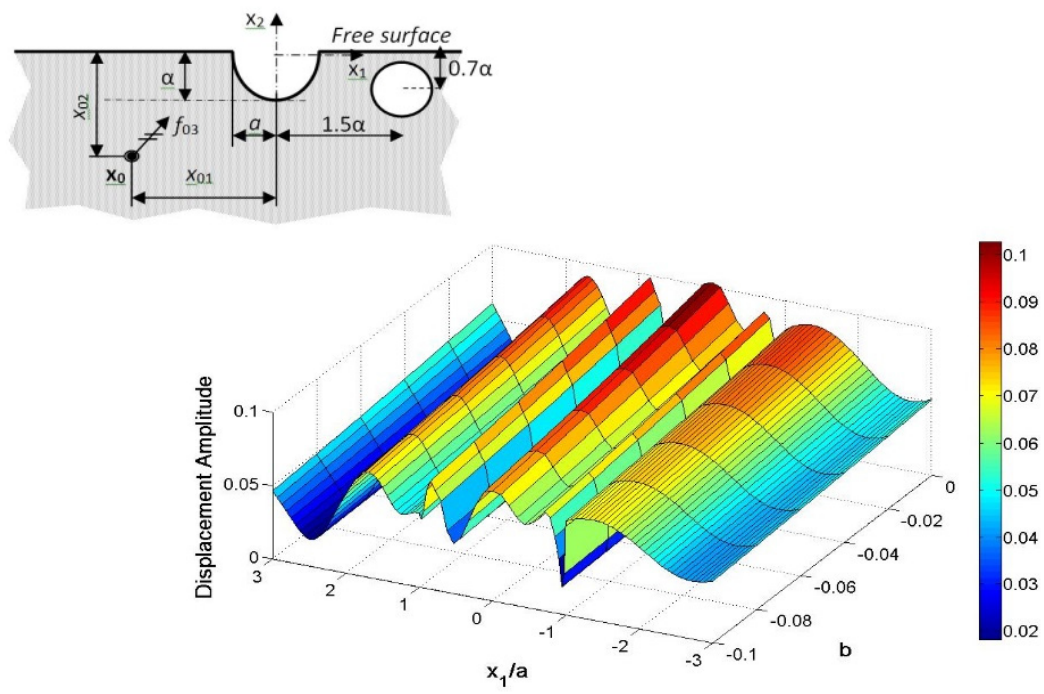

Figure 6: Displacement amplitude along the free surface of inhomogeneous half-plane with a semi-circular canyon and a circular cavity for $0<\mathrm{b}<-0.1$ subjected to waves radiating from an embedded seismic source at point $(0,-5 a)$ for $\eta=1.25$. 


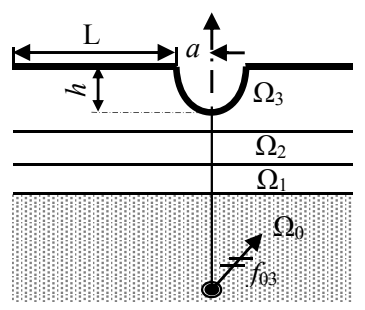

a)

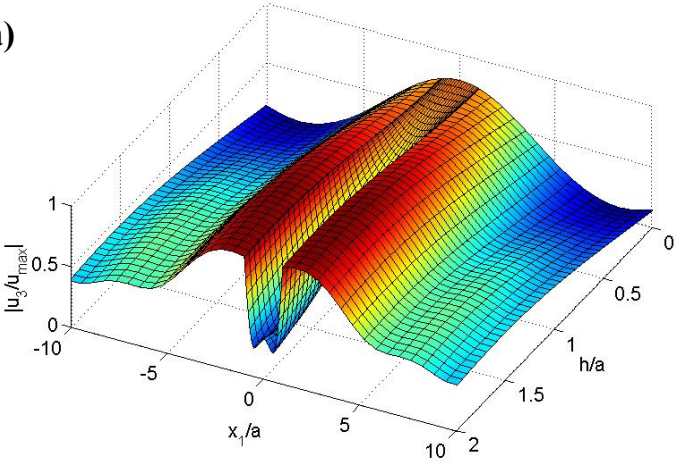

b)

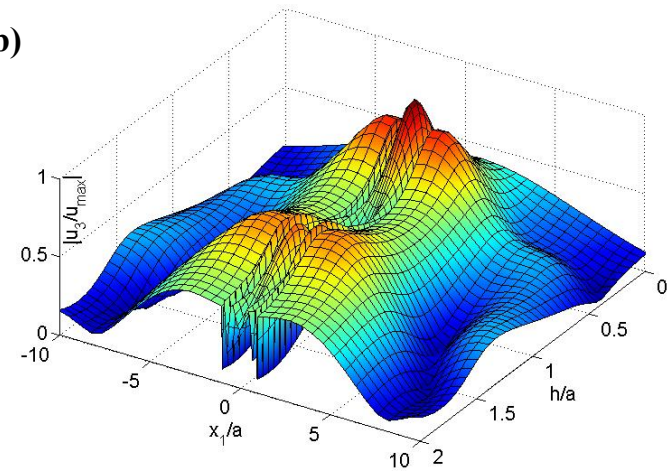

Figure 7: Normalized displacement amplitude along the free surface of a stratified half-plane with a semi-elliptic canyon having variable semi-axis $\mathrm{h}$ due to $\mathrm{SH}$ wave radiating from a line seismic source embedded at point $\mathrm{x}_{0}(0,5 \mathrm{a})$ for a fixed normalized frequency: (a) $\eta=0.25$; (b) $\eta=1$.

\section{Acknowledgements}

The authors acknowledge the support of the DFG under the grant number $\mathrm{Wu}$ 496/5-1. The second author (SLP) wishes to acknowledge the support provided through a Research and Design Centre of UACEG, Sofia grant No. BN 158/14. 


\section{References}

[1] Kennett BLN Seismic Wave Propagation in Stratified Media, Cambridge University Press, New York, 1983.

[2] Dominguez J., Boundary elements in dynamics, Computational Mechanics Publications, Southampton, 1993.

[3] Panza G, Paskaleva I, Dineva P, La Mura Cr., Earthquake site effects modelling by hybrid MS-BIEM: The case study of Sofia Bulgaria, Rendiconti di Scienze Fisiche by the Accademia dei Lincei 20: 91-116, 2009.

[4] Wuttke F, Dineva P, Schanz T., Seismic wave propagation in laterally inhomogeneous geological region via a new hybrid approach, Journal of Sound and Vibration 330: 664-684, 2011.

[5] Rangelov TV, Dineva PS., Steady-state plane wave propagation in inhomogeneous 3D media, J. Theor. Appl. Mech., 35: 17-38, 2005.

[6] Rangelov TV and Manolis GD. Point force and dipole solutions in the inhomogeneous half-plane under time-harmonic conditions, Mechanics Research Communications, 56: 90-97, 2013. 